\title{
Monitoring one-electron photo-oxidation of guanine in DNA crystals using ultrafast infrared spectroscopy
}

Article

Accepted Version

Hall, J. P., Poynton, F. E., Keane, P. M., Gurung, S. P., Brazier, J. A., Cardin, D. J., Winter, G., Gunnlaugsson, T., Sazanovich, I. V., Towrie, M., Cardin, C. J., Kelly, J. M. and Quinn, S. J. (2015) Monitoring one-electron photo-oxidation of guanine in DNA crystals using ultrafast infrared spectroscopy. Nature Chemistry, 7. pp. 961-967. ISSN 1755-4330 doi: https://doi.org/10.1038/nchem.2369 Available at https://centaur.reading.ac.uk/46101/

It is advisable to refer to the publisher's version if you intend to cite from the work. See Guidance on citing.

Published version at: http://www.nature.com/nchem/journal/vaop/ncurrent/full/nchem. 2369. html

To link to this article DOI: http://dx.doi.org/10.1038/nchem.2369

Publisher: Nature Publishing Group

All outputs in CentAUR are protected by Intellectual Property Rights law, including copyright law. Copyright and IPR is retained by the creators or other copyright holders. Terms and conditions for use of this material are defined in the End User Agreement. 


\section{CentAUR}

Central Archive at the University of Reading

Reading's research outputs online 


\section{Monitoring one-electron photo-oxidation of guanine in DNA crystals using ultrafast infrared spectroscopy}

James P. Hall, ${ }^{1,2}$ Fergus E. Poynton, ${ }^{3}$ Páraic M. Keane, ${ }^{1,3}$ Sarah P. Gurung, ${ }^{1,2}$ John A. Brazier, ${ }^{4}$ David J. Cardin, ${ }^{1}$ Graeme Winter, ${ }^{2}$ Thorfinnur Gunnlaugsson, ${ }^{3}$ Igor V. Sazanovich, ${ }^{5}$ Michael Towrie, ${ }^{5}$ Christine J. Cardin, ${ }^{1}$ John M. Kelly ${ }^{3}$ and Susan J. Quinn ${ }^{6}$

${ }^{1}$ Department of Chemistry, University of Reading, Whiteknights, Reading RG6 6AD, United Kingdom

${ }^{2}$ Diamond Light Source, Harwell Science and Innovation Campus, Didcot, Oxfordshire, OX11 0QX

${ }^{3}$ School of Chemistry, Trinity College, University of Dublin, Dublin 2, Ireland

${ }^{4}$ Department of Pharmacy, University of Reading, Whiteknights, Reading RG6 6AD, United Kingdom

${ }^{5}$ Central Laser Facility, Research Complex at Harwell, Science \& Technology Facilities Council, Rutherford Appleton Laboratory, Didcot, Oxfordshire, OX11 0QX, UK

${ }^{6}$ School of Chemistry, University College Dublin, Dublin 4, Ireland

Email: christinejcardin@gmail.com,jmkelly@tcd.ie,susan.quinn@ucd.ie

Abstract: Photosensitised DNA-damage typically proceeds by one-electron photo-oxidation of guanine. The precise dynamics of this process are sensitive to the location and the orientation of the photosensitiser on the DNA, which are very difficult to define in solution. To overcome this, ultrafast time-resolved infrared (TRIR) spectroscopy was performed on photo-excited ruthenium polypyridyl/DNA crystals whose atomic structure has been determined by X-ray crystallography. Combining the X-ray and TRIR data we are able to define both the geometry of the reaction site and the rates of individual steps in a reversible photo-induced electron transfer process. This allows us to propose an individual guanine as the reaction site and intriguingly reveals that dynamics in the crystal state are quite similar to that observed in the solvent medium.

Knowing the mechanism and dynamics of photo-induced DNA damage is critically important for our understanding of diseases associated with UV-irradiation as well as the development of photosensitised DNA-directed therapies. ${ }^{1,2}$ In particular, there is considerable interest in developing photo-activated metal-based DNA-targeted drugs, which avoid the severe side effects of metal based chelation therapies such as those from the cisplatin family. ${ }^{1}$ To this end ruthenium polypyridyl complexes are excellent photosensitiser candidates as the ligand structure facilitates both the binding to DNA and the desired photo-damage through oxidation and/or adduct formation. ${ }^{2-5}$ The principal target of this approach is usually the modification of guanine, the most susceptible nucleic acid base, providing a route to biological deactivation. The extent of photosensitised guanine oxidation is determined by the yield and lifetime of the initially formed electron transfer products, which is determined by the rates of the forward and back electron transfer. ${ }^{6}$ These rates are controlled by a number of factors which include the binding site of the photosensitiser, the redox 
potential of the closest nucleobase and the spatial arrangement of the photosensitiser in the binding site. Our understanding of these processes has been greatly advanced through the use of timeresolved spectroscopy. ${ }^{7}$ In particular, time-resolved infra-red (TRIR) has emerged as a powerful tool to characterise the evolution of DNA damage as it yields functional group information as well as producing kinetic information. Examples include the identification of precursors to pyrimidine dimer formation ${ }^{8}$ and infrared marker bands for the radical cations of guanine $e^{9,10}$ and methyl cytosine. ${ }^{10}$ Two-dimensional TRIR methods have also been used to probe the role of the water sheath of DNA, for example in protein binding interactions. ${ }^{11}$

To date the interpretation of data obtained using steady state and time-resolved spectroscopy has been limited by a lack of precise knowledge of the spatial arrangement of the photosensitising metal complex at the binding site. However, we recently reported the atomic resolution crystal structure of a ruthenium photosensitiser bound to DNA, specifically $\Lambda$ - $\left[\operatorname{Ru}(\operatorname{TAP})_{2}(\mathrm{dppz})\right]^{2+}(\operatorname{TAP}=1,4,5,8$ tetraazaphenanthrene and dppz = dipyrido[3,2-a:2',3'-c]phenazine) bound to (TCGGCGCCGA) ${ }_{2}{ }^{12}$ and subsequently, crystal structures have also been obtained for $\Lambda$-[Ru(phen $\left.)_{2}(\mathrm{dppz})\right]^{2+}$ and $\Delta$-[Ru(bpy $\left.)_{2}(\mathrm{dppz})\right]^{2+}$ bound to other DNA sequences. ${ }^{13,14}$ The availability of these crystals permits us for the first time to examine the ultrafast electron transfer processes leading to the oxidation of guanine in systems where the molecular arrangement of the relevant reactive species is precisely known. Note also that this study is performed at high concentrations, which are comparable to the molecularly crowded environment that drugs encounter in the cell, where macromolecules constitute up to $40 \%(\mathrm{w} / \mathrm{v}) .{ }^{15}$ It also allows us to ascertain how the dynamics in solution and in the more crowded and static crystal environment differ.

The direct probing of a crystal sample is non-trivial as it requires the use of conditions that avoid crystal damage and that permit transmission of the probe light. The crystal system we have chosen $\left.\left\{\Lambda-\left[R u(T A P)_{2}(d p p z)\right]^{2+} \text { bound to (TCGGCGCCGA }\right)_{2}\right\},^{12}$ has already been shown by atomic resolution $X-$ ray diffraction to be robust (for example at room temperature it can be reversibly dehydrated and rehydrated, even though this causes large movements of the nucleic acid). ${ }^{16}$ In our study here we use micron size crystal fragments, which ensures sufficient transmission of the probe IR light. 


\section{Results and Discussion}

Herein we report a TRIR spectroscopic study of nucleic acids in a crystal. In particular, we describe the ultrafast electron transfer between a ruthenium polypyridyl complex and a DNA decamer sequence (Fig 1a). Crystals of $\Lambda$ - $\left[\operatorname{Ru}(T A P)_{2}(d p p z)\right]^{2+}$ bound to (TCGGCGCCGA) $)_{2}$ were grown from a crystallization solution containing $\mathrm{H}_{2} \mathrm{O}$ using the previously reported procedure. ${ }^{12}$ As with other macromolecular crystals, there is a high water content, whose vibrations absorb strongly in the infrared window where DNA nucleobases appear $\left(1400-1700 \mathrm{~cm}^{-1}\right)$. For this reason a $\mathrm{D}_{2} \mathrm{O}$ exchange was performed by soaking the crystal in a $\mathrm{D}_{2} \mathrm{O}$ solution and crystallographic data were recorded, to a final outer shell resolution of $1.21 \AA$ (Supplementary Table 1, Fig 1b). This dataset showed the structure to be fully consistent with that previously reported for the $\mathrm{H}_{2} \mathrm{O}$ equilibrated crystal (Fig 1cf). ${ }^{12}$ In the crystal structure, the smallest repeating unit (the crystallographic asymmetric unit) is a single strand of DNA and a single ruthenium complex. Thus there is only one environment for a ruthenium complex. The intercalation cavity is closed on the 'pyrimidine side' $\left(T_{1}-C_{2}\right)$, but on the 'purine side' the terminal adenine $A_{10}$ stacks onto a symmetry related metal complex, forming a reverse Watson-Crick basepair with $T_{1}$ (Fig 1f). Since the DNA backbone plays no part in the electron transfer process, the difference between this arrangement and that of the corresponding closed intercalation cavity ${ }^{13}$ is just that of the terminal purine base orientation. The ground state infrared spectrum of the crystals recorded before and after exchange is found to be dominated by characteristic nucleobase vibrations especially those of guanine and cytosine (Supplementary Fig. S4).
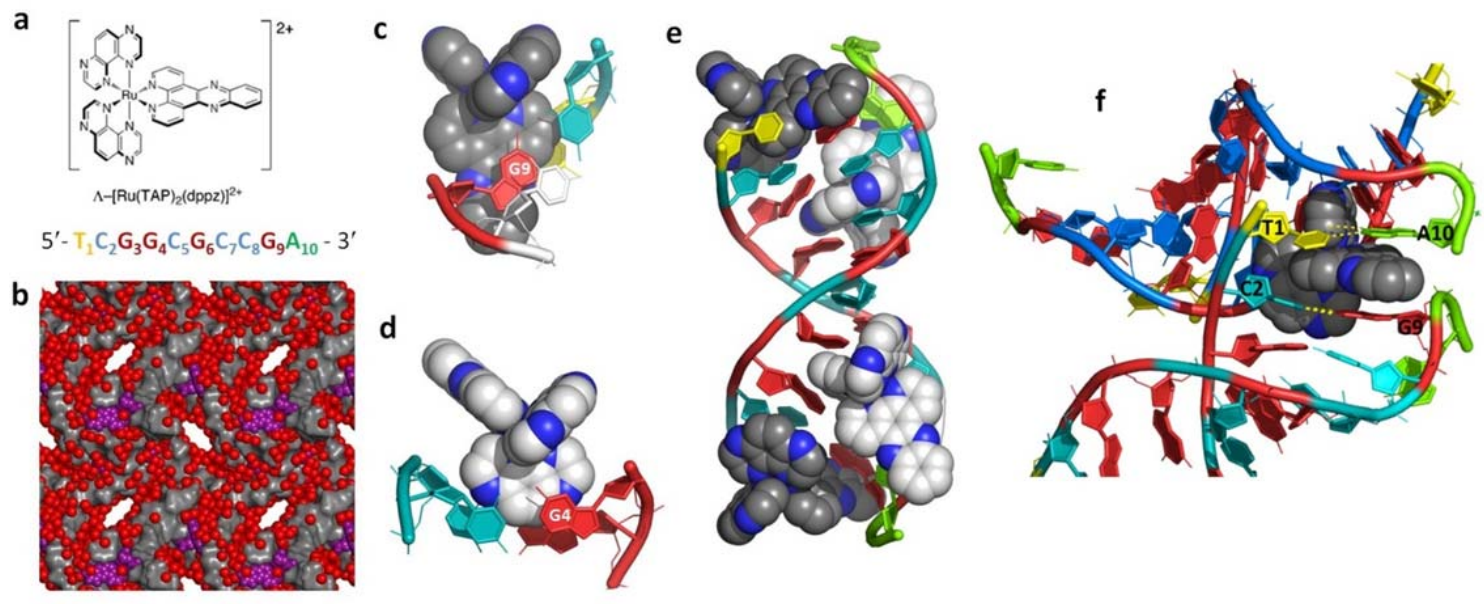

Figure 1 Crystal structure in the presence of $D_{2} \mathbf{O}$. $a$, Structure of $\Lambda$ - $\left[R u(T A P)_{2}(d p p z)\right]^{2+}$ and the DNA sequence used, which has been colour coded according to base type. $\mathbf{b}$, Illustration of the extended crystal lattice. $\mathrm{D}_{2} \mathrm{O}$ molecules are drawn as red spheres, DNA in grey and complexes in purple. c, The terminal intercalation site showing the complex intercalated, through the dppz group, into the $G_{9} A_{10}: T_{1} C_{2}$ step. $d$, The complex semi-intercalates, through the TAP ligand, into the $\mathrm{G}_{3} \mathrm{G}_{4}: \mathrm{C}_{7} \mathrm{C}_{8}$ step, inducing a kink into the helix. e, the structure of the duplex with all four interacting complexes. $\mathbf{f}$, Four symmetry equivalent strands of the decamer in the crystal structure, to show the environment of the ruthenium complexes. The $\mathrm{A}_{10}$ is seen to flip out to form a reverse Watson-Crick base pair with $\mathrm{T}_{1}$ from a neighbouring duplex, completing the intercalation cavity. All complexes are symmetry equivalent and cross-link the duplexes in the crystal. Colour code; Nitrogen atoms in the complex are coloured blue, $T=$ yellow, $G=$ red, $A=$ green, $C=$ cyan for $\mathbf{c}-\mathbf{e}$ while for $\mathbf{f} C$ = cyan on the duplex containing the $C_{2}$ of the intercalation cavity and $C=$ marine blue on the second duplex. 
Transient infrared spectroscopic measurements were recorded for the $\mathrm{D}_{2} \mathrm{O}$-exchanged crystals using the ULTRA instrument at the Central Laser Facility, STFC, configured to allow measurement in the picosecond to microsecond time range. ${ }^{17,18}$ The pump-probe experimental setup is shown in Fig. 2 a. In this arrangement hundreds of individual crystal fragments (approximate size, 2.5-5 $\mu \mathrm{m}$, obtained by crushing the as-grown crystals which are approximately $150 \times 150 \times 100 \mu \mathrm{m}$ ) are sandwiched between two $\mathrm{CaF}_{2}$ plates held in place using a demountable liquid cell, Fig. 2a. The crystals are suspended in an exchange solution containing 35\% (v/v) 2-methyl-2,4-pentanediol (MPD) in either $\mathrm{H}_{2} \mathrm{O}$ or $\mathrm{D}_{2} \mathrm{O}$ which acts to maintain hydration.

a
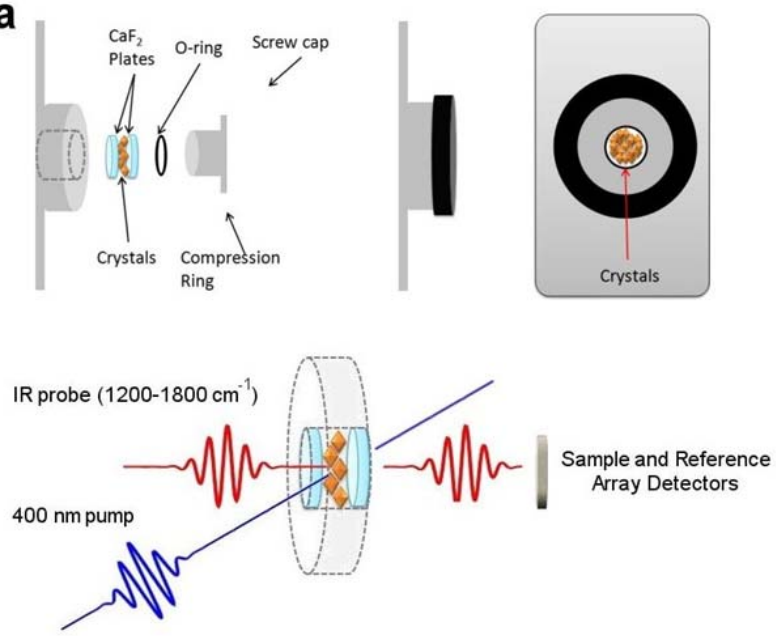

b
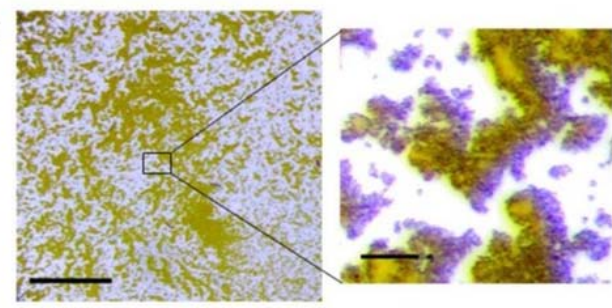

C
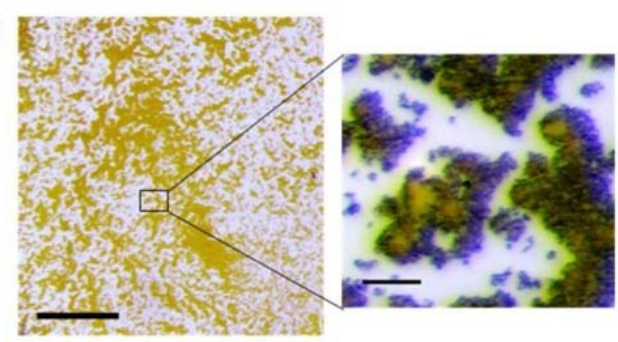

Figure 2 Experimental scheme and microscopy controls for the time-resolved infra-red spectroscopy experiments on crystal samples. a, Experimental set-up. The crystal sample is held between two $\mathrm{CaF}_{2}$ plates. The metal complex is selectively excited using a $400 \mathrm{~nm}(50 \mathrm{fs}, 1 \mu \mathrm{J}, 1 \mathrm{kHz})$ pulse and the infrared spectrum of the complex and DNA is recorded at different time delays. $\mathbf{b}$, Crystals positioned between two $\mathrm{CaF}_{2}$ plates before irradiation. $\mathbf{c}$, Absence of visible damage to crystals after irradiation. Scale bars: left $1300 \mu \mathrm{m}$, right $70 \mu \mathrm{m}$ (The blue colour is due to polarisation affects.)

To minimise photodamage of the sample the demountable cell was placed under continuous rapid raster. In this configuration the crystals were excited at $400 \mathrm{~nm}(50 \mathrm{fs}, 1 \mu \mathrm{J}, 1 \mathrm{kHz}$ ) and probed in the mid-infrared window $(5.7-8.3 \mu \mathrm{m})$ with spectra recorded at a range of time delays. Rigorous checks were performed to ensure that sample integrity was maintained during the experiment. No evidence of crystal damage or melting was observed when microscope images recorded before and after irradiation were compared (Fig. 2b-c).

Highly structured TRIR spectra for the crystal samples were recorded at regular intervals from 2 picoseconds out to 10 microseconds after laser excitation of the metal complex (a selection of these are shown in Fig. 3a-c). Between 1250 and $1545 \mathrm{~cm}^{-1}$ the spectrum is dominated by the transient species originating from the metal complex (Supplementary Fig 5). The TRIR spectrum shortly after excitation (20 ps) shows that the excited state possesses a strong transient broad absorption band at $1456 \mathrm{~cm}^{-1}$ as well as a number of sharp transient bands and a particularly well resolved negative-going 'bleach' at $1275 \mathrm{~cm}^{-1}$, due to depletion of the ground state (Fig. 3a). At wavenumbers greater than $1545 \mathrm{~cm}^{-1}$ transient features are observed due to changes in the ground state vibrations of the DNA bases (Fig. 3a). In particular four strong DNA bleaches at $1680 \mathrm{~cm}^{-1}$ ( $\mathrm{C}=\mathrm{O}$ of guanine), $1645 \mathrm{~cm}^{-1}$ ( $\mathrm{C}=\mathrm{O}$ of cytosine), $1620 \mathrm{~cm}^{-1}$ (ring vib. of adenine) and $1580 \mathrm{~cm}^{-1}$ (ring vib. guanine) may be identified..$^{19}$ The presence of these bands, in the absence of DNA direct excitation has been observed 
in a number of previous TRIR studies of metal complex intercalating systems ${ }^{6,20-24}$ and is attributed to a perturbation of groundstate vibrations due to an electrostatic (Stark) effect ${ }^{25-26}$ that arises because of changes in the charge distribution of the neighbouring molecule upon excitation. ${ }^{26-29}$ As this effect is known to be localised (typically $<6 \AA$ ) the bleached bands therefore report on the immediate surroundings of the excited state. ${ }^{25-29}$

These changes can be correlated with the crystal structure as follows. There are two distinct guanine-containing sites:- (1) The $G_{9}$ base is stacked onto the central pyrazine ring of the intercalated dppz ligand at the terminal $T_{1} C_{2}: G_{9} A_{10}$ step (Fig. 1C). (2) One of the two TAP ligands is wedged into the $\mathrm{G}_{3} \mathrm{G}_{4}: \mathrm{C}_{7} \mathrm{C}_{8}$ step in a semi-intercalated (kinking) mode (Fig. 1d). In addition, the purine ring of $A_{10}$ overlaps strongly with the pyrazine rings of the dppz (Fig. 1e) and this presumably is the cause of the strong adenine bleach observed. Interestingly, while the $\mathrm{D}_{2} \mathrm{O}$ solution TRIR data for the bound complex also reveals bleach bands due to the carbonyl stretches, in contrast to the crystal data, the adenine bleach observed in solution is weak (Supplementary Fig 6). The adenine absorbance in the groundstate FTIR spectra of the crystal and the solution are found to be quite similar (Supplementary Fig 7) and so the difference observed in the TRIR spectra may indicate that the complex intercalates at other base-pair steps in solution. ${ }^{20}$
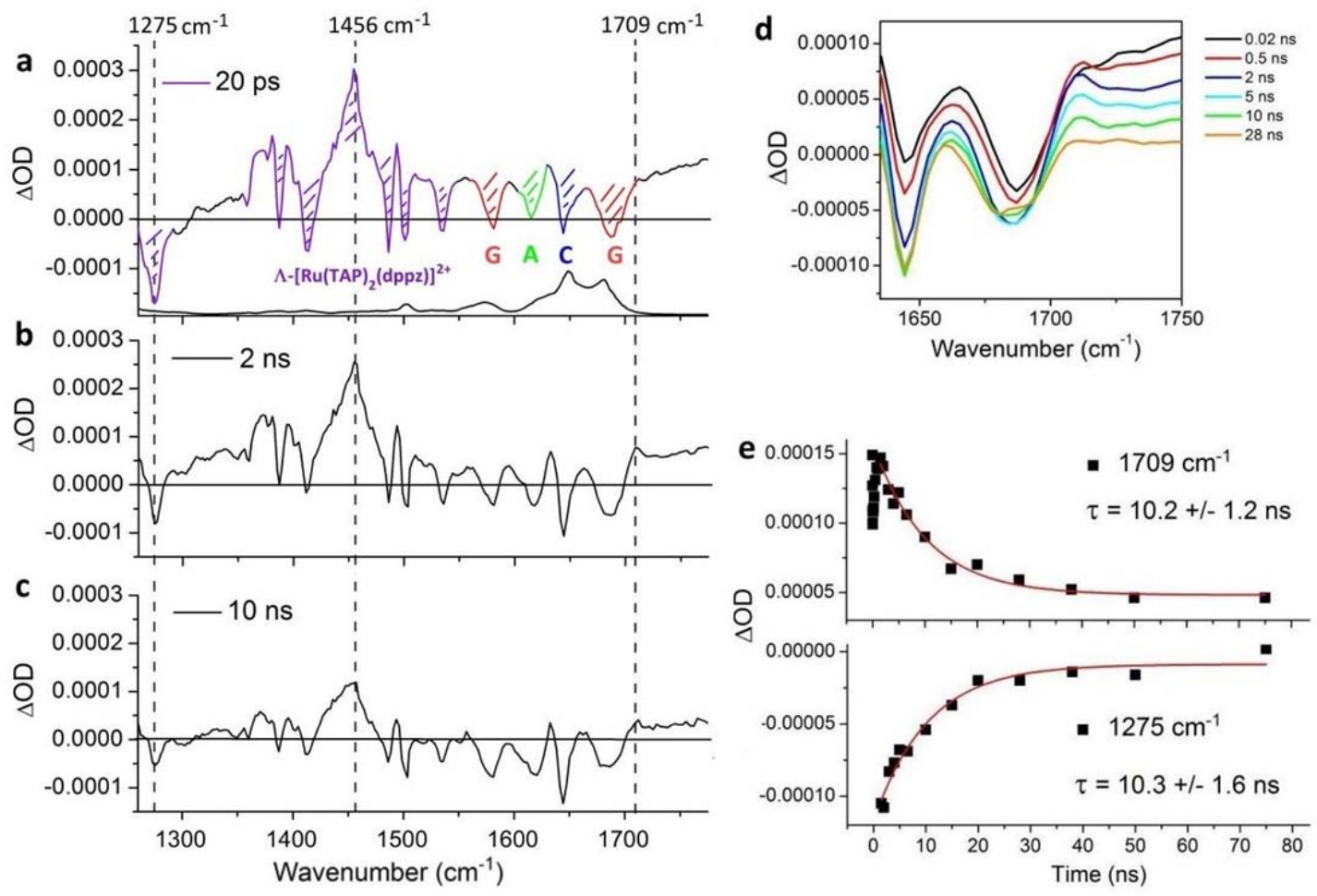

Figure 3 Transient infrared absorption spectra obtained from $D_{2}$ O-exchanged crystal samples of $\Lambda$-[Ru(TAP) $\left.)_{2}(\mathrm{dppz})\right]^{2+}$ bound to (TCGGCGCCGA) $)_{2}$. a-c, TRIR spectra after $20 \mathrm{ps}, 2 \mathrm{~ns}$ and $10 \mathrm{~ns}$ following $400 \mathrm{~nm}$ ( $50 \mathrm{fs}, 1 \mu \mathrm{J}, 1 \mathrm{kHz}$ ) excitation (ground state FTIR included in a). d Spectral evolution of the GC carbonyl band region. e Monoexponenetial kinetic profiles of the $\Lambda$ - $\left[\mathrm{Ru}(\mathrm{TAP})_{2}(\mathrm{dppz})\right]^{2+}$ species $\left(1275 \mathrm{~cm}^{-1}\right)$ and the guanine radical cation $\left(1709 \mathrm{~cm}^{-1}\right.$, see Fig S8). 
Further changes occur between 20 ps and 2 ns, during which time the recorded difference spectra change as the excited state of $\Lambda$-[Ru(TAP) $\left.)_{2}(d p p z)\right]^{2+}$ is reduced to form $\Lambda$-[Rull(TAP)(TAP $\left.\left.{ }^{*}\right)(\mathrm{dppz})\right]^{+}$ and the DNA is oxidised. In particular, the bleach structure of the $C$ and $G$ bands are found to change and an additional absorption band at $1709 \mathrm{~cm}^{-1}$ is observed (Fig 3a-c). The formation of these species from the excited state occurs on the picosecond time domain with a rate constant for the forward reaction of $1 / 500 \mathrm{ps}^{-1}$ (See Supplementary Fig. 7). The band at $1709 \mathrm{~cm}^{-1}$ has previously been assigned to the guanine radical cation by comparing DFT calculations with the experimental spectrum at $77 \mathrm{~K}$ in glass. ${ }^{9}$ In that study the DFT calculation for the cation assumed a value of 1.231 $\AA$ for the guanine $\mathrm{C}-\mathrm{O}$ bond distance in the radical. This length is close to that observed for the relevant 9-guanine $\mathrm{C}-\mathrm{O}$ ground state double bond distance in this crystal (1.233 $\AA$, compared to the restrained distance of $1.237 \AA$ ), which suggests that the formation of the guanine radical cation should not perturb the local structure of the crystal. The reformation of the ground state by the back electron transfer reaction between the one-electron oxidised guanine and the reduced excited state of the metal complex is readily followed by monitoring the TRIR signals at $1275 \mathrm{~cm}^{-1}$ and $1709 \mathrm{~cm}^{-1}$ (Fig 3e) which yield a rate constant for the back reaction of $1 / 10 \mathrm{~ns}^{-1}$, see scheme $1 . \%$ Similar TRIR features to those observed for the crystal sample have been obtained for studies of the complex in the presence of the guanine-containing DNA sequences in $\mathrm{D}_{2} \mathrm{O}$ buffered solution, while complementary solution visible transient absorption measurements $s^{s}$ also indicate the formation of the reduced metal complex excited state, $\Lambda$-[Ru $\left.{ }^{\prime \prime}(\operatorname{TAP})\left(\operatorname{TAP}^{0^{-}}\right)(\mathrm{dppz})\right]^{+}{ }^{6,20}$ Efficient reversible oxidation has also been proposed in cases where reduced mobility of the intercalated dppz ligand prevents access to the geometry needed to form a photo-product with the TAP ligand. ${ }^{30}$

Table 1: Measured lifetimes for the forward and reverse electron transfer between $\Lambda-\left[R u(T A P)_{2}(d p p z)\right]^{2+}$ and $(\text { TCGGCGCCGA })_{2}$ in the crystal state ${ }^{a}$ Baseline corrections have been applied (see Supplementary Fig 8).

\begin{tabular}{|c|c|c|c|c|}
\hline & \multicolumn{2}{|c|}{$\begin{array}{c}\Lambda-\left[\mathrm{Ru}(\mathrm{TAP})_{2}(\mathrm{dppz})\right]^{2+} \\
\text { Bleach }\left(1275 \mathrm{~cm}^{-1}\right) \text { and Transient }\left(1456 \mathrm{~cm}^{-1}\right)\end{array}$} & \multicolumn{2}{|c|}{ Transient $\left(1709 \mathrm{~cm}^{-1}\right)$} \\
\hline & reverse & reverse & forward & reverse \\
\hline $\mathrm{D}_{2} \mathrm{O}$ crystal & $10.3 \pm 1.6 \mathrm{~ns}$ & $10 \pm 1.0 \mathrm{~ns}$ & $500 \pm 80$ ps & $10 \pm 1.2 \mathrm{~ns}$ \\
\hline $\mathrm{H}_{2} \mathrm{O}$ crystal & $12.5 \pm 0.8 \mathrm{~ns}$ & $8.4 \pm 1.0 \mathrm{~ns}$ & $278 \pm 30 \mathrm{ps}$ & $9.0 \pm 0.4 \mathrm{~ns}$ \\
\hline
\end{tabular}

These experiments demonstrate that the excited state of $\Lambda$-[Ru(TAP $\left.)_{2}(\mathrm{dppz})\right]^{2+}$ in the crystal can cause reversible one-electron oxidation of guanine in DNA. However, the ground state crystal structure indicates that three separate guanine bases $\left(G_{3}, G_{4}\right.$ and $\left.G_{9}\right)$ interact with the complex and are therefore the candidates for photo-oxidation, see Fig. 4a. The distance between the complex and the guanine is an important factor in photo-induced electron transfer processes. ${ }^{30}$ As shown in Fig. $4 \mathrm{a}-\mathrm{c}$, the $2-\mathrm{ND}_{2}$ groups of all three guanines are quite close to the ruthenium metal atom (distances between the 2-ND $\mathrm{ND}_{2}$ nitrogen of $\mathrm{G}_{3}, \mathrm{G}_{4}$ and $\mathrm{G}_{9}$ from the ruthenium atom are 6.6, 5.3 and $4.6 \AA$ respectively). However, the greatest overlap of the metal complex is with $G_{9}$, which is substantially stacked on the pyrazine ring (the degree of stacking of the guanine ring systems is approximately $85 \%$, see Supplementary Fig 10 ). The mean of the two perpendicular distances between the pyrazine $\mathrm{N}$ atoms and the guanine base plane was calculated as $3.32 \AA$ (since the dppz plane and the guanine 
plane are not exactly parallel). This distance is that expected for the separation of interacting $\pi$ systems in DNA, and these pi-pi interactions may be significant for the electron transfer.

a

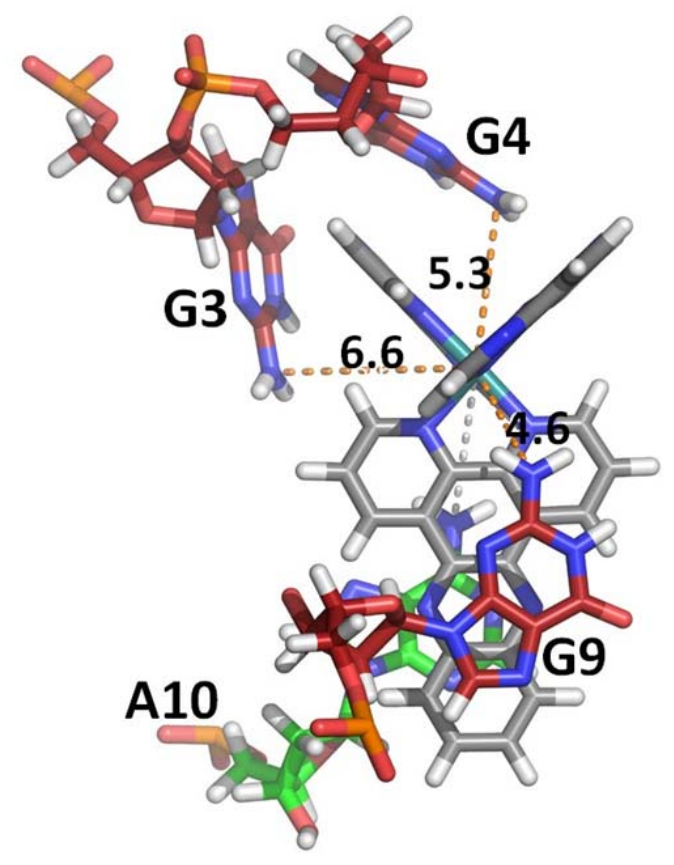

b
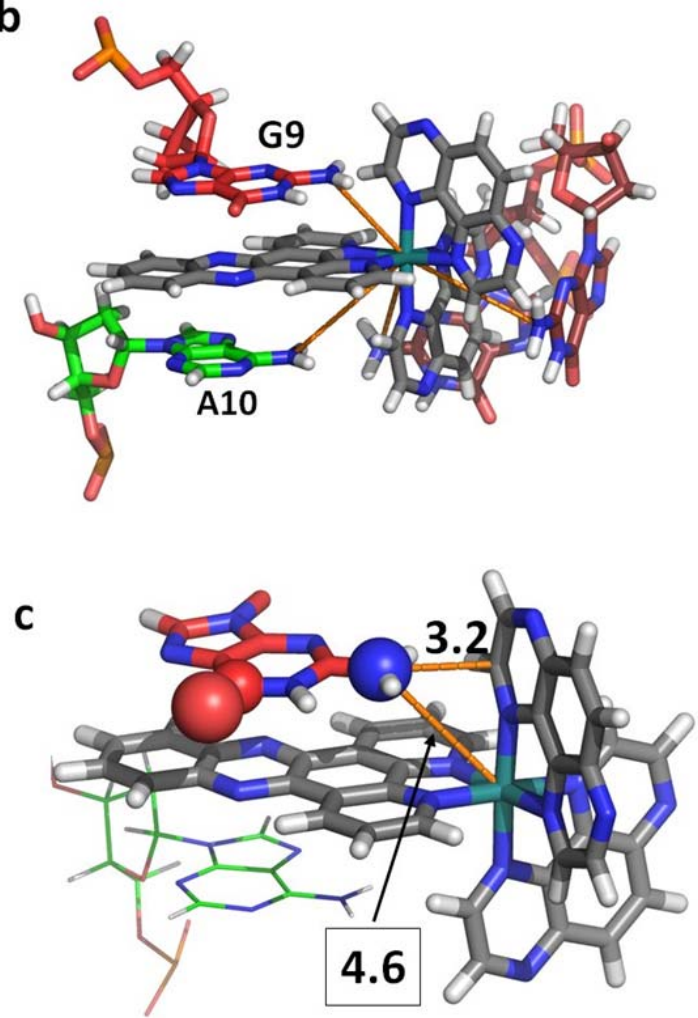

Figure $4 \mid$ Proposal for the site of reversible electron transfer from guanine to $\Lambda$ - $\left[\mathrm{Ru}(\mathrm{TAP})_{2}(\mathrm{dppz})\right]^{2+}$

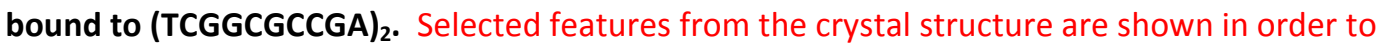
propose the site and mechanism. a Three guanine residues surround the ruthenium complex, $G_{3}, G_{4}$ and $G_{9} . G_{3}$ and $G_{4}$ define the semi-intercalation site of one TAP ligand, and $G_{9}$ and $A_{10}$ the intercalation site of the dppz ligand with Ru-N2 distances shown ( $\AA$ ). $\mathbf{b}$ The same model reoriented to show the $G_{9}$ and $A_{10}$ geometries with respect to the ruthenium atom and the dppz ligand. The orange dashed lines link the $2 N$ of $G_{9}$ and the $6 \mathrm{~N}$ of $A_{10}$ to the ruthenium atom, with $A_{10}$ in the reverse Watson-Crick geometry which brings the 6 -amino group to $5.6 \AA$ from the ruthenium. $A$ plausible mechanism for guanine oxidation is via the amino group of $G_{9} \mathbf{c} A$ zoom to show the close approach of the second TAP ligand to the $G_{9}$ guanine amino group (the $N$ shown as a small sphere, with the closest approach to the nearest carbon only $3.2 \AA$ while the centroid distance is $3.6 \AA$ ). $A$ plausible mechanism for the back-reaction is via electron transfer from the TAP. The 6-carbonyl group which monitors the reversible oxidation of guanine is also shown as small spheres. Colour scheme - Ruthenium - teal; nitrogen - blue; oxygen - bright red; carbon - green for $A_{10}$, red for $G_{9}$, dark red for $\mathrm{G}_{3}$ and $\mathrm{G}_{4}$, grey for dppz and TAP ligands to ruthenium.

This arrangement of the complex leads us to propose that, as illustrated in Fig. 4b and Fig. 4c, the primary electron transfer site is from the $G_{9}$ in the intercalation cavity. This allows us to build up a comprehensive picture of the electron transfer event in the crystal sample, see Scheme 1 . An 
important question is whether this picture has any bearing on solution phenomena. The rate constant for the back reaction $\left(1 / 10 \mathrm{~ns}^{-1}\right)$ is not markedly different from that found in $\mathrm{D}_{2} \mathrm{O}$ solutions of the same deoxyoligonucleotide $\left(1 / 17 \mathrm{~ns}^{-1}\right)$, where we expect that there will be a variety of intercalation sites in the deoxyoligonucleotide, each with different rates of decay. Sensitivity to the intercalation site is reflected in the different rate constants observed for alternating $\mathrm{GC}$ (i.e. $(\mathrm{GC})_{5}$ ) $\left(1 / 12 \mathrm{~ns}^{-1}\right)$ and contiguous $G\left(\mathrm{G}_{5} \mathrm{C}_{5}\right)\left(1 / 5.5 \mathrm{~ns}^{-1}\right) .{ }^{20}$ This observation is intriguing as it might be expected that the dynamics would be significantly different in the crystal state where structural flexibility such as base breathing are absent. This suggests that the dominating factor for the electron transfer process is intercalation at a G-containing basepair. ${ }^{21}$

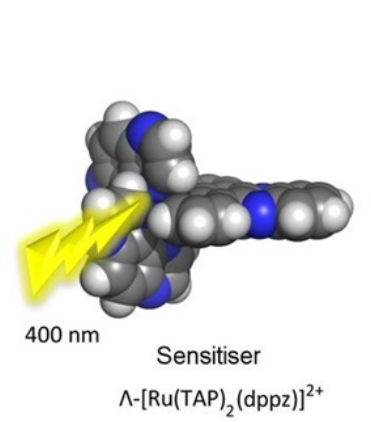

$\mathrm{T}_{1}-\mathrm{C}_{2}-\mathrm{G}_{3}-\mathrm{G}_{4}-\mathrm{C}_{5} \mathrm{G}_{-} \mathrm{C}_{-}-\mathrm{C}_{\overline{8}} \mathrm{G}_{9}-\mathrm{A}_{10}$ A-G-C-C-G-C-G-G-C-T

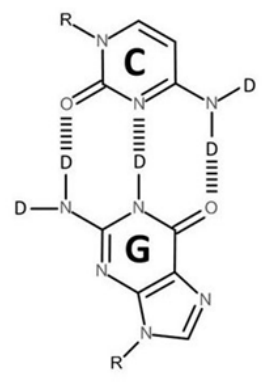

$A-\left[\operatorname{Ru}(\mathrm{TAP})_{2}(\mathrm{dppz})\right]^{2}$

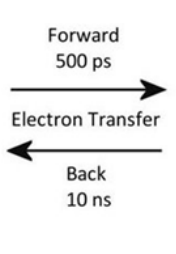

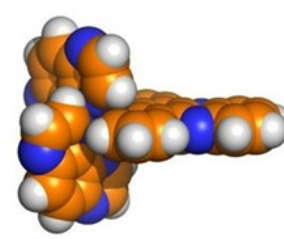

$\Lambda-\left[\operatorname{Ru}\left(\operatorname{TAP}^{-}\left(\mathrm{TAP}^{\bullet-}\right)(\mathrm{dppz})\right]^{+}\right.$

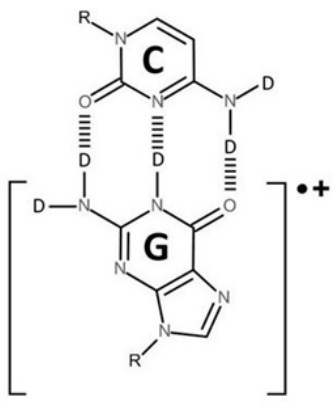

$\mathrm{T}_{1}-\mathrm{C}_{2}-\mathrm{G}_{3}-\mathrm{G}_{4}-\mathrm{C}_{5}-\mathrm{G}_{6}-\mathrm{C}_{7}-\mathrm{C}_{\overline{8}}^{-} \mathrm{G}_{9}^{-1} \mathrm{~A}_{10}$ A-G-C-C-G-C-G-G-C-T

\section{Scheme 1 Summary of reversible oxidation of the guanine site in $\Lambda-\left[\operatorname{Ru}(T A P)_{2}(d p p z)\right]^{2+}$ bound (TCGGCGCCGA) .}

In addition, TRIR spectra were also obtained for measurements carried out on crystals that had not undergone $\mathrm{D}_{2} \mathrm{O}$ exchange, see Table 1 . As expected the band structure of the complex was not significantly affected by the presence of $\mathrm{H}_{2} \mathrm{O}$ but laser-induced heating of the crystal sample cause a significant perturbation of the spectrum for the $\mathrm{H}_{2} \mathrm{O}$ vibrations between 1600 and $1700 \mathrm{~cm}^{-1}$ (See Supplementary Fig. 9). This is due to the temperature dependent absorbance of $\mathrm{H}_{2} \mathrm{O}$ and reflects the transition from fully hydrogen-bonded to partially hydrogen-bonded structures. ${ }^{31}$ While the presence of this strong feature obscures the key DNA bleaches, the transient band assigned to the guanine radical cation at $1712 \mathrm{~cm}^{-1}$ is more clearly visible due to the shift in the position of the guanine bleach to lower wavenumbers in the $\mathrm{H}_{2} \mathrm{O}$-equilibrated sample. The rate of the forward reaction is about twice as fast as in the $\mathrm{D}_{2} \mathrm{O}$-treated crystal, possibly a consequence of protoncoupling in the excited state electron transfer process. ${ }^{6}$ By contrast similar rates are observed in both $\mathrm{H}_{2} \mathrm{O}$ - and $\mathrm{D}_{2} \mathrm{O}$-equilibrated crystals for the reverse process. This ability to monitor biological systems in both environments may also prove useful for other proton-coupled processes.

\section{Conclusion}

The combination of TRIR spectroscopy and atomic resolution X-ray crystallography has offered additional insight that would have been impossible with either technique in isolation. In particular, it allows us to define both the geometry of the reaction site and the rates of individual steps in a reversible photoinduced electron transfer process. Importantly, this allows us to propose an individual guanine as the reaction site and more significantly, reveals that dynamics in the crystal state are quite similar to that observed in the solvent medium. We believe that this approach will 
also be useful for the study of electron transfer or other processes in biomolecules such as proteins. ${ }^{32}$ It may also be noted that in contrast to time resolved X-ray techniques such as those performed at FEL sources, which require hundreds of thousands of crystals, ${ }^{33-34}$ ultrafast spectroscopy is widely available in research laboratories with orders of magnitude less material required. This last point is particularly relevant for biological samples.

\section{Methods}

Crystals of $d\left(\right.$ TCGGCGCCGA) with $\Lambda$ - $\left[\operatorname{Ru}(T A P)_{2}(d p p z)\right]^{2+}$ were grown according to the previously given protocol. ${ }^{6}$ A crystal was soaked in a solution of $35 \%$ MPD $(v / v)$ in $D_{2} O$ for 30 minutes and was then flash cooled in liquid nitrogen. Data were collected on beamline 103 at Diamond Light Source and were processed using xia2. ${ }^{35}$ The structure was solved using the anomalous scattering of Barium with SHELXC/D/E. ${ }^{36}$ The model was built using $\operatorname{Coot}^{37}$ and refined in REFMAC. ${ }^{38}$ For full details of the structure solution and refinement can see Supplementary Table 1. TRIR measurements were carried out in the Time Resolved Multiple Probe, TRMPS, mode of the instrument. ${ }^{11}$ The mid IR probe was generated by difference frequency conversion of the signal and idler output of a $10 \mathrm{kHz}, 8 \mathrm{~W}, 40 \mathrm{fs}$, $800 \mathrm{~nm}$ titanium sapphire laser pumped optical parametric amplifier and the pump by second harmonic of a $1 \mathrm{kHz}, 4 \mathrm{~W}, 120 \mathrm{fs}, 800 \mathrm{~nm}$ titanium sapphire laser. ${ }^{17,18}$

\section{Footnotes}

$\$$ Note that visible transient absorption spectra cannot be carried out with micron-sized crystals because (a) the excessively high absorbance of the sample distorts the signal and (b) the light is highly scattered. This scattering effect is orders of magnitude less in the mid IR where the average crystal size is smaller than the wavelength of the light. This makes TRIR especially applicable to samples of this dimension.

\% Similar lifetimes are recorded at $1456 \mathrm{~cm}^{-1}$ as $\Lambda-\left[\operatorname{Ru}^{\prime \prime}(\operatorname{TAP})\left(\operatorname{TAP}^{0^{-}}\right)(\mathrm{dppz})\right]^{+}$reoxidises. Note that a very broad band present at $1437 \mathrm{~cm}^{-1}$ (Fig. 3d) may be attributed to the $\delta$ bending mode of HOD due to temperature jump of small quantities of insufficiently exchanged $\mathrm{H}_{2} \mathrm{O}$ in the crystal. This band, as well as some other bleached bands, is found to persist at longer times (see Supplementary Fig 10).

Supplementary Information is linked to the online version of the paper at www.nature.com/naturechemistry

Acknowledgements The work was supported by BBSRC grants BB/K019279/1 and BB/M004635/1 (to CJC, JAB, MT and JPH) and a Royal Irish Academy /Royal Society International Exchange Scheme award (to CJC, JMK and TG). These experiments were carried out thanks to programmed access approved by the CLF (Application No. 14230014). The authors gratefully acknowledge the contribution of Hanna Beer and Katrin Buchner to the preparation of the metal complex used in this study. The authors would also like to thank the University of Reading for the provision of the Chemical Analysis Facility. SJQ acknowledges support from the College of Science, UCD. GW would like to thank Katherine McAuley for provision of in-house beam time on I03. We would like to thank Thomas Sorensen (Diamond Light Source), Juan Sanchez-Weatherby (Diamond Light Source) and Susana Teixeira (Institut Laue-Langevin and University of Keele) for useful discussions. 


\section{References}

1. Smith, N.A. \& Sadler P.J., Photoactivatable metal complexes: from theory to applications in biotechnology and medicine Phil. Trans R. Soc.A., 371, 20120519, (2013).

2. Gill, M. R. \& J. A. Thomas, Ruthenium(ii) polypyridyl complexes and DNA from structural probes to cellular imaging and therapeutics. Chem. Soc. Rev. 41, 3179-3192 (2012).

3. Puckett C.A. \& Barton J.K., Methods to Explore Cellular Uptake of Ruthenium Complexes, J. Am. Chem. Soc., 129, 46-47, (2007).

4. Marcélis L., Moucheron C. \& Kirsch-De Mesmaeker, A., Ru-TAP complexes and DNA: from photo-induced electron transfer to gene photo-silencing in living cells. Phil. Trans. R. Soc. A, 371, 20120131, (2013).

5. Ryan, G.J., Quinn S. \& Gunnlaugsson T., Highly Effective DNA Photocleavage by Novel "Rigid" $\mathrm{Ru}(\mathrm{bpy})_{3}$-4-nitro- and -4-amino-1,8-naphthalimide Conjugates Inorg. Chem. 47, 401-403 (2008).

6. Elias, B. et al. Photooxidation of guanine by a ruthenium dipyridophenazine complex intercalated in a double-stranded polynucleotide monitored directly by picosecond visible and infrared transient absorption spectroscopy. Chem. Eur. J., 14, 369-75, (2008).

7. Towrie, M. , Doorley, G. W., George, M. W., Parker, A. W., Quinn, S. J. \& Kelly, J. M. ps-TRIR covers all the bases - recent advances in the use of transient IR for the detection of short-lived species in nucleic acids. Analyst 134, 1265-1273 (2009).

8. Schreier W. J. et al. Thymine dimerization in DNA is an ultrafast photoreaction. Science 315, 625-629 (2007).

9. Parker, A. W., Lin, C. Y., George, M. W., Towrie, M. \& Kuimova, M. K. Infrared characterization of the guanine radical cation: finger printing DNA damage. J. Phys Chem. B. 114, 3660-3667, (2010).

10. Bucher, D. B., Pilles, B. M., Carell T. \& Zinth, W. Charge separation and charge delocalization identified in long-living states of photoexcited DNA. Proc. Natl. Acad. Sci. (USA) 111, 43694374 (2014).

11. Khesbak, H., Savchuk, O., Tsushima, S. \& Fahmy, K. The role of water H-bond imbalances in B-DNA substate transitions and peptide recognition revealed by time-resolved FTIR spectroscopy J. Am. Chem. Soc. 133, 5834-5842 (2011).

12. Hall, J. P. et al. Structure determination of an intercalating ruthenium dipyridophenazine complex which kinks DNA by semiintercalation of a tetraazaphenanthrene ligand. Proc. Natl. Acad. Sci (USA) 108, 17610-17614 (2011).

13. Niyazi H., et al. Crystal structures of $\Lambda$ - $\left[\mathrm{Ru}(\mathrm{phen})_{2} \mathrm{dppz}\right]^{2+}$ with oligonucleotides containing TA/TA and AT/AT steps show two intercalation modes. Nat. Chem. 4, 621-628 (2012).

14. Song H., Kaiser J. T., \& Barton J. K. Crystal structure of $\Delta-R u(b p y)_{2} \mathrm{dppz}^{2+}$ bound to mismatched DNA reveals side-by-side metalloinsertion and intercalation Nat. Chem. 4, 615-620 (2013).

15. Zimmerman S. B. \& Trach, S. O. Estimation of macromolecule concentrations and excluded volume effects for the cytoplasm of Escherichia coli. J Mol Biol. 222, 599-620 (1991).

16. Hall, J. P. et al. Controlled dehydration of a ruthenium complex-DNA crystal induces reversible DNA kinking. J. Am. Chem. Soc. 136, 17505-17512 (2014).

17. Greetham, G.M. et al. ULTRA: A unique instrument for time-resolved spectroscopy. Appl. Spectrosc. 64, 1311 (2010). 
18. Greetham, G. M.; Sole, D.; Clark, I. P.; Parker, A. W.; Pollard, M. R.; Towrie, M. Time-resolved multiple probe spectroscopy. Rev. Sci. Instrum. 83, 103107 (2012).

19. Banyay, M., Sarkar, M. \& Gräslund, A. Infrared library of nucleic acids. Biophys Chem. 104, 477-88 (2003).

20. Keane, P. M. et al. Enantiomeric conformation controls rate and yield of photo-induced electron transfer in DNA sensitized by Ru(II) dipyridophenazine complexes. J. Phys. Chem. Lett. 6, 734-738 (2015).

21. Keane, P.M. et al. Reversal of a Single Base-Pair Step Controls Guanine Photo-Oxidation by an Intercalating Ruthenium(II) Dipyridophenazine Complex. Angew. Chem. Intl. Ed. DOI: 10.1002/ange.201502608 (2015).

22. Devereux S.J. et al. Study of picosecond processes of an intercalated dipyridophenazine $\mathrm{Cr}$ (III) complex bound to defined sequence DNAs using transient absorption and time-resolved infrared methods Dalton Trans. 43, 17606-17609 (2014).

23. Olmon, E.D. et. al. Charge Photoinjection in Intercalated and Covalently Bound $\left[\operatorname{Re}(\mathrm{CO})_{3}(\mathrm{dppz})(\mathrm{py})\right]^{+}-$DNA Constructs Monitored by Time-Resolved Visible and Infrared Spectroscopy J. Am. Chem. Soc., 133, pp 13718-13730 (2011).

24. Smith, J.A., George, M.W. \& Kelly J.M. Transient Spectroscopy of Dipyridophenazine Metal Complexes which undergo electron transfer with DNA Coord. Chem Rev. 255, 2666-2675 (2011).

25. Park E.S. \& Boxer, S.G. Origins of the Sensitivity of Molecular Vibrations to Electric Fields: Carbonyl and Nitrosyl Stretches in Model Compounds and Proteins J. Phys. Chem. B, 106, 5800- 5806 (2002).

26. Fried S.D. \& Boxer, S.G. Boxer Measuring Electric Fields and Noncovalent Interactions Using the Vibrational Stark Effect Acc. Chem. Res. 48, 998-1006 (2015).

27. Volk, M. et. al. Peptide Conformational Dynamics and Vibrational Stark Effects Following Photoinitiated Disulfide Cleavage J. Phys. Chem. B, 101, 8607-8616 (1997).

28. Volk, M. et al. Carbonyl Spectator Bonds as Sensitive Sensors for Charge Transfer Reactions on the Femtosecond Time Scale J. Phys. Chem. A, 104, 4984-4988 (2000).

29. Movsisyan, L.D., Peeks, M.D., Greetham, G.M. Towrie, M. Thompson, A.L., Parker, A.W. \& Anderson H.L., Photophysics of Threaded sp-Carbon Chains: The Polyyne is a Sink for Singlet and Triplet Excitation. J. Am. Chem. Soc. 136, 17996-18008 (2014).

30. Le Gac S. et al. Photo-reactive Rull-oligonucleotide conjugates: influence of an intercalating ligand on the inter- and intra-strand photo-ligation processes. Dalton Trans. 39, 9672-9683 (2010).

31. Walrafen, G.E., Hokmabadi, M.S. \& Yang, W.H. Raman investigation of the temperature dependence of the bending $\mathrm{v} 2$ and combination $\mathrm{v} 2+\mathrm{vL}$ bands from liquid water J. Phys. Chem. 92, 2433-2438 (1988).

32. Shih, C. et al. Tryptophan-Accelerated Electron Flow Through Proteins. Science 320, $1760-$ 1762 (2008).

33. Kupitz, C., et al. Serial time-resolved crystallography of photosystem II using a femtosecond Xray laser. Nature 513, 261-265 (2014).

34. Tenboer, J. et al. Time-resolved serial crystallography captures high-resolution intermediates of photoactive yellow protein. Science 346, 1242-1246 (2014).

35. Winter, G. xia2:an expert system for macromolecular crystallography data reduction. J. Appl. Crystallogr. 43, 186-190 (2010). 
36. Sheldrick, G.M. A short history of SHELX. Acta. Crystallogr. A64, 112-122 (2008).

37. Emsley, P., Lohkamp, B., Scott, W.G. \& Cowtan, K. Features and development of Coot. Acta. Crystallogr, D66, 486-501 (2010).

38. Murshudov, G.N., Vagin, A.A. \& Dodson, E.J. Refinement of macromolecular structures by the maximum-likelihood method. Acta. Crystallogr. D53, 240-255 (1997). 